\title{
Electron Energy Loss Spectroscopy Study on the Dielectric Response of Single
}

\section{$\mathrm{H}_{2} \mathrm{Ti}_{3} \mathrm{O}_{7}$ Nanotube}

J. Wang ${ }^{*}$, Q. Li ${ }^{*}$ L.-M. Peng ${ }^{* *}$ and M. Malac ${ }^{* * *}$

*Department of Physics, The Chinese University of Hong Kong, Shatin, New Territory, Hong Kong **Key Laboratory on the Physics and Chemistry of Nanodevices and Department of Electronics, Peking University, Beijing 100871, China

***National Institute for Nanotechnology, 11421 Saskatachewan Drive, Edmonton Alberta, Canada T6G 2M9

Tubular nanostructured materials have aroused significant research interest due to their promising properties associated with the small size, large surface to volume ratio and specific geometrical configuration [1]. For example, $\mathrm{TiO}_{2}$ nanotubes are found to possess high photocatalytic activity comparing to their bulk materials [2], leading to potential applications in photocatalysis devices, environmental purification and gas sensors. As an analog with the $\mathrm{TiO}_{2}$ nanotubes, $\mathrm{H}_{2} \mathrm{Ti}_{3} \mathrm{O}_{7}$ nanotubes with the diameter less than 10nm (Fig. 1) exhibit similar band gap with $\mathrm{TiO}_{2}$ and can be simply synthesized by alkali treatment of commercial $\mathrm{TiO}_{2}$ powder [3]. However, more detailed electronic structure and the dielectric response properties of individual nanotube are still not known.

To investigate the electronic structure and the dielectric response properties of individual $\mathrm{H}_{2} \mathrm{Ti}_{3} \mathrm{O}_{7}$ nanotube, the spatially resolved electron energy loss spectroscopy (SREELS) study is carried out in both real space and momentum transfer space on single nanotube, the results are compared to that of $\mathrm{TiO}_{2}$. The SREELS technique is found to be superior to the conventional line scan mode of the scanning transmission electron microscopy (STEM) in current experiment. As parallel electron beam is employed in the SREELS, it is able to provide better momentum transfer resolution and introduce less electron irradiation damage to the sensitive sample.

The momentum transfer resolved EELS study (Fig. 2 (a)) shows that the $\mathrm{H}_{2} \mathrm{Ti}_{3} \mathrm{O}_{7}$ nanotube has similar basic electronic structure (including the band gap, single electron interband transitions and collective oscillation of the free electrons) to that of $\mathrm{TiO}_{2}$, except the additional defect states in the band gap of $\mathrm{H}_{2} \mathrm{Ti}_{3} \mathrm{O}_{7}$ nanotube. On the other hand, several surface related features are found to be dominant in the zero momentum transferred EEL spectrum of single nanotube and their intensity dramatically decrease in larger momentum transfer. The surface related nature of those excitations are further confirmed by the spatially resolved EELS carried out in real space (Fig. 2 (b)) and they are probably attribute to the different surface modes (in-plane and out-of-plane modes) due to the surface coupling in anisotropic nanostructure [4].

References:

[1] R. Tenne, L. Margulis, M. Genut, and G. Hodes, Nature (London) 360, 444 (1992)

[2] M. Adachi, Y. Murata, M. Harada, S. Yoshikawa, Chem. Lett. 8, 942 (2000)

[3] Q. Chen, W. Zhou, G. H. Du, and L.-M. Peng, Adv. Mater. 14, 1208 (2002) 
[4] M. Kociak, O. Stéphan, L. Henrard, V. Charbois, A. Rothschild, R. Tenne, and C. Colliex, Phys. Rev. Lett. 87, 075501 (2001)

[5] This work is supported by grants from the research grant council of HKSAR (project No. CUHK 402105) and direct grant (2060317). Additional support was obtained from NINT and NSERC.
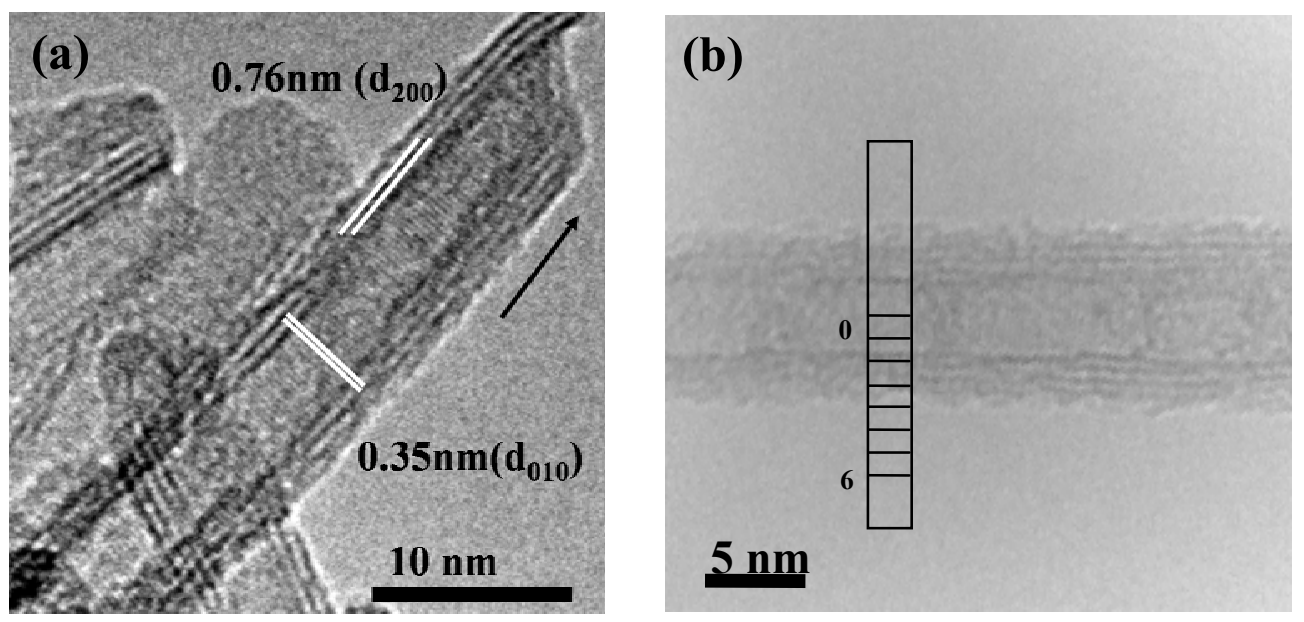

Figure 1. (a) High resolution TEM image of the $\mathrm{H}_{2} \mathrm{Ti}_{3} \mathrm{O}_{7}$ nanotubes and (b) Illustration of the sample region examined in SREELS experiment.
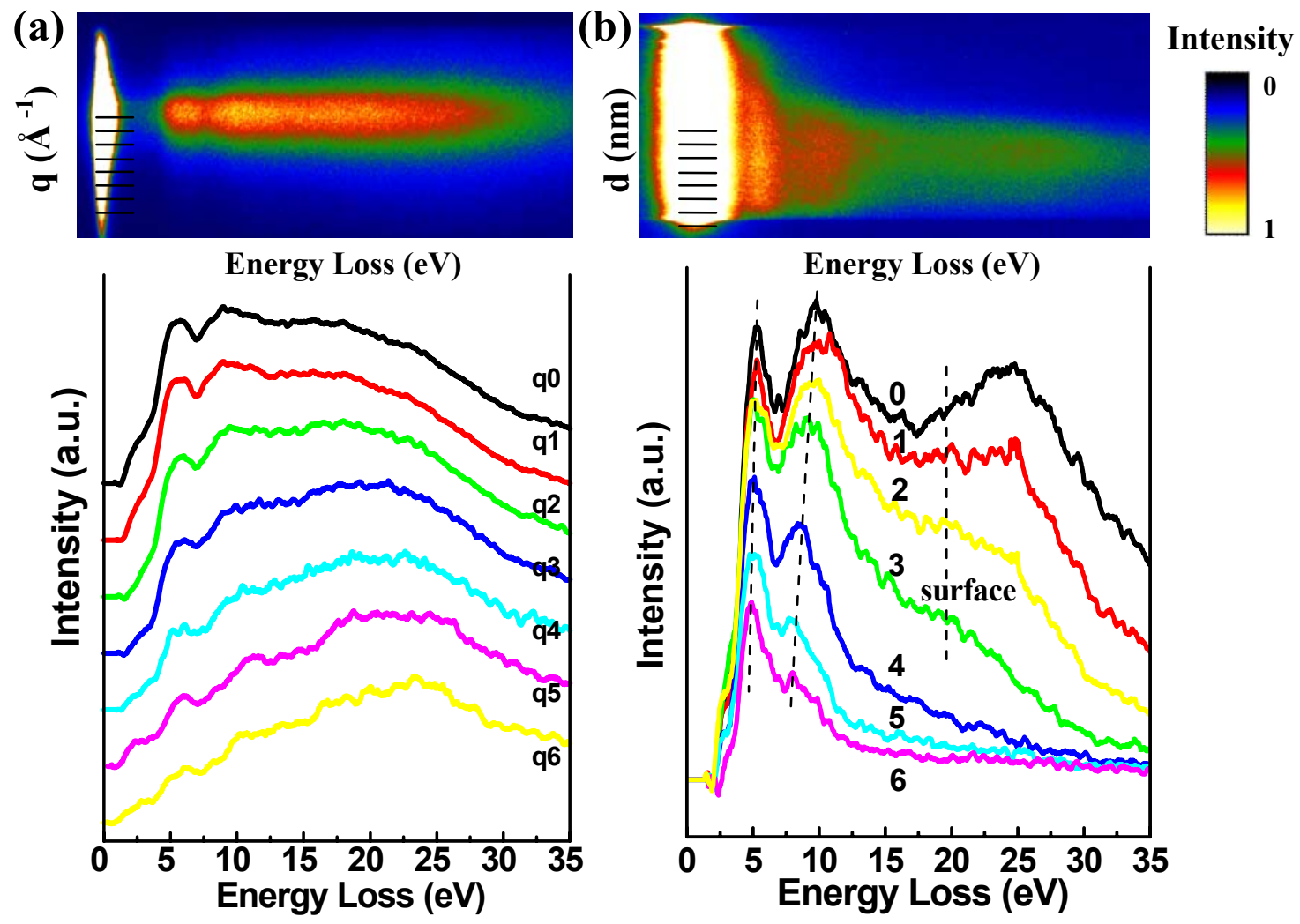

Figure 2. (a) The momentum transfer resolved SREELS image of individual $\mathrm{H}_{2} \mathrm{Ti}_{3} \mathrm{O}_{7}$ nanotube and the EEL spectra extracted from $\mathrm{q} 0=0$ to $\mathrm{q} 6=0.4 \AA^{-1}$. (b) SREELS image of individual $\mathrm{H}_{2} \mathrm{Ti}_{3} \mathrm{O}_{7}$ nanotube in image mode and spectra extracted from position 0 to 6 (as shown in Fig 1 (b)). 